\section{Nueva A ntropología}

Nueva Antropología

ISSN: 0185-0636

nuevaantropologia@hotmail.com

Asociación Nueva Antropología A.C.

México

Figueroa Perea, Juan Guillermo; Rivera Reyes, Gabriela Algunas reflexiones sobre la representación social de la sexualidad femenina Nueva Antropología, vol. XII, núm. 41, marzo, 1992, pp. 101-121

Asociación Nueva Antropología A.C.

Distrito Federal, México

Disponible en: http://www.redalyc.org/articulo.oa?id=15904107

- Cómo citar el artículo

- Número completo

- Más información del artículo

Página de la revista en redalyc.org

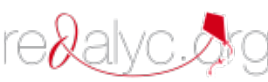

Sistema de Información Científica

Red de Revistas Científicas de América Latina, el Caribe, España y Portugal Proyecto académico sin fines de lucro, desarrollado bajo la iniciativa de acceso abierto 


\title{
Algunas reflexiones sobre la representación social de la sexualidad femenina*
}

\author{
Juan Guillermo Figueroa Perea y \\ Gabriela Rivera Reyes**
}

\section{INTRODUCGION}

La sexualidad femenina ha sido, durante años, foco de polémicas y discusiones. En torno al cuerpo de la mujer se entretejen mitos, discursos científicos y planteamientos ideológicos. La complejidad y riqueza del tema ha dado lugar a una proliferación de estudios al respecto. El tra-

* Trabajo presentado con motivo de la "Reunión latinoamericana de antropología de la mujer", organizada dentro del Programa Interdisciplinario de Estudios de la Mujer de El Colegio de México, en junio de 1990. La encuesta que sirve de base para este trabajo fue financiada por la Organización Mundial de la Salud (OMS), a través de su Programa de Investigación en Reproducción Humana.

** Juan Guillermo Figueroa, filosofo; Gabriela Rivera Reyes, psicóloga. Investigadores de la Dirección General de Planificación Familias de la Secretarta de Salud. bajo que presentamos en esta ocasión pretende aportar información reciente sobre este tema, y trata de responder a dos interrogantes: ¿qué opina la mujer acerca de las decisiones sobre su sexualidad y reproducción?, y ¿qué significados y valores deposita en las relaciones sexuales?

Nos interesa estudiar la sexualidad femenina, a través de la representación social que de ella se tiene, y relacionar esta representación con algunas características socioeconómicas y demográficas. No pretendemos una exposición acabada del estudio de la representación social de la sexualidad femenina, sino un primer acercamiento al tema, a partir de la información generada por la Encuesta sobre Determinantes de la Práctica Anticonceptiva en México (EDEPAM). Dentro del marco 
conceptual de esta encuesta, la representación de la sexualidad forma parte del grupo de variables culturales que inciden en la práctica anticonceptiva. El interés principal de la EDEPAM no es específicamente la representación de la sexualidad, sino el estudio de las variables que determinan la dinámica del uso de métodos anticonceptivos en México. No obstante, la riqueza de la información generada en dicha investigación permite el tipo de análisis como el que vamos a desarrollar.

Este tipo de aproximación analítica -a través de la representación social-representa una posibilidad más de interpretar la información obtenida por una encuesta demografica en hogares, una posibilidad que no centra su atención en la práctica anticonceptiva o en factores demográficos, sino en explorar un poco algunos elementos culturales a partir de un acercamiento a las representaciones sociales. Hemos decidido no poner el acento en la práctica anticonceptiva sino en la sexualidad, pues se piensa que resulta más pertinente de acuerdo al tema de esta mesa. En la primera parte del trabajo expondremos una breve semblanza de la EDEPAM; posteriormente, trataremos de ubicar el concepto de representación social en su relación con la cultura, y, en la última parte, abordaremos la representación de la sexualidad en la mujer a partir de dos líneas de análisis: a) la apropiación del cuerpo femenino en términos de la toma de decisiones, que lleva a cabo la mujer sobre su sexualidad y su reproducción; y b) la representación social de la sexualidad en su relación con algunas características socioeconómicas, demográficas y culturales.

\section{BREVE DESCRIPCION DE LA EDEPAM}

Los estudios demográficos han señalado la existencia de limitaciones, en cuanto a la explicación de los factores que determinan el uso de métodos anticonceptivos. Se han detectado las variables demográficas que ejercen influencia considerable sobre la práctica anticonceptiva y el comportamiento reproductivo en nuestro país. Sin embargo, poco se ha hecho acerca de los determinantes culturales que ejercen influencia en la regulación de la fecundidad. Una de las investigaciones que ha hecho un esfuerzo por integrar las variables culturales al estudio de la regulación de la fecundidad es la EDEPAM: puede considerarse como un estudio de carácter fundamentalmente demográfico, con una muestra que pretende ser representativa de tres regiones del país, pero incorporando inquietudes de tipo antropológico.

Para el diseño de este estudio se tomó como punto de partida la información generada por la Encuesta $\mathrm{Na}$ cional sobre Fecundidad y Salud (EN. FEs) de 1987 (ver Figueroa et al., 1988). La EDEPAM constituye un seguimiento de algunas mujeres entrevistadas en la ENFES, y de las que ya se tenía información sobre sus caracte- 
rísticas socioeconómicas y demográficas. La EDEPAM se aplicó a 1487 mujeres de edad fértil (entre 15 y 49 años), y que en su historia de vida hubieran tenido la experiencia de haber estado alguna vez unidas.

La EDEPAM se realizó en 1988, en tres regiones de la República Mexicana que presentan cierta heterogeneidad en cuanto a sus características socioeconómicas, demográficas y culturales:

Región I.Noroeste: Baja California Norte, Baja California Sur, Sinaloa, Sonora y Nayarit.

Región II. Bajío: Guanajuato, Hidalgo y Querétaro.

Región III. Sureste: Chiapas, Campeche, Tabasco, Quintana Roo y Yucatán.

En la EDEPAM se explora una serie de variables demogrâficas, sociocconómicas y culturales. El instrumento para la recopilación de información fue un cuestionario integrado por 12 secciones. En la última sección se indaga acerca de la valoración atribuida a la maternidad, y acerca de las opiniones y valoración de las relaciones sexuales.

Ahora bien, por una parte nos ubicamos en un estudio que pretende analizar variables culturales $y$, por otra, decimos que tratamos de acercarnos a la sexualidad femenina desde la perspectiva de su representación social; cabe entonces hacer algunas aclaraciones sobre la relación que existe entre cultura y representación social.

\section{CULTURA Y REPRESENTACION SOCIAL}

En las últimas décadas los estudios sobre representación social han cobrado gran importancia dentro de la psicología social. Dentro del campo de la representación, no se pretenden las explicaciones universales, sino que se retoman y se replantean los diferentes objetos de estudio de la psicología en relación con las condiciones sociales que les dan origen. Existen varios autores que se han dedicado al estudio de la representación; como antecedentes podemos ubicar a Durkheim y a Freud; posteriormente dos de los autores que han dado grandes avances en este terreno han sido Serge Moscovici y René Kaes.

\section{El concepto de representación social}

Para el desarrollo de este trabajo, entendemos por representación social el conjunto de opiniones y creencias, y la organización de las percepciones, valores y conocimientos relativos a determinados aspectos del mundo del individuo. De ninguna manera afirmamos la necesidad de un proceso racional, sino de una combinación de elementos a través de los cuales nos ponemos en contacto con la realidad de la que formamos parte.

Este concepto tiene sus orígenes en la filosofia y la psicología clásicas; se utilizó para designar el contenido de un acto de pensamiento. La re- 
presentación se define como social por excelencia: no existe ser social sin representación. En este sentido la representación no se refiere sólo al contenido sino al proceso de la actividad mental de la realidad, fungiendo como mediadora entre procesos perceptivos y cognoscitivos, y dan por resultado una imagen (objeto representado) que es lo que tiende a convertirse en real.

Moscovici define la representación como un universo de opiniones. R. Kaes adopta esta definición y la completa con la adición del têrmino "creencias", entendiendo éstas como "la organizacion duradera de las percepciones y conocimientos relativos a un cierto aspecto del mundo del individuo". La representación es una respuesta simbolica que modula la conducta del sujeto. Para Herzlich la representación social implica una actividad de reproducción de las propiedades de un objeto, que se lleva a cabo al nivel concreto, metafórico, y que se organiza en torno a una significación central. La representación es, entonces, una construcción mental del objeto, inseparable de la actividad simbólica de un sujeto. La representación no es un reflejo mecánico de la realidad, sino la articulación entre lo psíquico y lo social, entre las actividades perceptivas, $\operatorname{cog}$ noscitivas y las afectivas. La representación, como respuesta simbólica, desempeña una función fundamental en la comunicación colectiva, para ser más precisos, en la generación y transmisión de saberes colectivos y en las conductas sociales.

La representación emerge a raíz de determinadas condiciones, pero se constituye, no como un mero reflejo de las mismas, sino como "algo más", recreando la realidad en el universo simbolico del individuo, y se materializa en un conjunto de opiniones, creencias, valores y conductas. La red de representaciones colectivas ordenadas con las funciones sociales de comunicación, intercambio, identificación y transformación, constituye la cultura.

\section{SEXUALIDAD, REPRESENTACION Y CULTURA}

Del conjunto de representaciones sociales, nos interesa específicamente la representación social de la sexualidad. Al tratar de estudiar la sexualidad, nos encontramos que lo que se dice de ella está revestido de ideología, de mitos y secretos, y de prohibiciones; pero también de concesiones, de discursos diversos y mecanismos ambiguos que giran en torno a la sexualidad.

En el sentido más amplio, la sexualidad se refiere, no sólo a las actividades dependientes del aparato genital sino a una serie de excitaciones y actividades presentes desde el inicio del desarrollo psicobiológico. En este sentido, la sexualidad no constituye un dispositivo ya estructurado previamente, sino que se va estableciendo a lo largo de la historia individual, 
y se constituye en el seno de estructuras intersubjetivas. Suponemos que hay opiniones, valores y elementos afectivos atribuidos a la sexualidad, y que esta esfera de componentes refleja factores ideológicos y culturales.

Dentro de la amplia gama de componentes que engloba el concepto de sexualidad, hemos decidido situarnos en el terreno de las relaciones sexuales. La elección de este aspecto de la sexualidad y no de otro, responde a la intención en la EDEPAM de relacionarlo con la práctica anticonceptiva, en la medida que parece ser el más cercano al suceso de la reproducción (y la anticoncepción).

\section{REPRESENTACION DE LA SEXUALIDAD EN LA MUJER}

Existen distintos planteamientos teóricos que desarrollan la manera cómo se percibe socialmente la sexualidad femenina. Uno de los planteamientos más interesantes en este sentido ha sido el desarrollado por Franca Basaglia. ${ }^{1}$ Esta autora señala que el cuerpo femenino ha sido considerado como "cuerpo para otros"; plantea que las fases de la historia de la mujer pasan por las modificaciones y alteraciones de su cuerpo. La preservación y custodia del núcleo familiar, el embarazo y la función materna han marcado

1 Basaglia, F., "La mujer y la locura", Antipsiquiatra y política, Extemporáneos, 1978. el cuerpo de la mujer como un "cuerpo para otros": para la procreación o para el goce del hombre. Basaglia plantea que incluso el hecho de la penetración durante el acto sexual se ha interpretado culturalmente como apropiación o expropiación.

La carencia de poder, señala $\mathrm{Zi}$ llah Eisenstein, ${ }^{2}$ que padece la mujer en nuestra sociedad, está basada en cuatro estructuras básicas: la de producción, la de reproducción, la sexualidad y la socialización de los niños. De estas cuatro estructuras, la dimensión de la sexualidad femenina ha sido en donde existen más prohibiciones, según los planteamientos de Juliet Mitchell. ${ }^{3}$

Ahora bien, si analizamos la producción discursiva acerca de la sexualidad femenina, encontramos que no sólo hay prohibiciones, sino que también existen -como mencionamos con anterioridad-concesiones y planteamientos ambigüos: La exageración de las dotes femeninas de seducción, tan en boga actualmente en las imágenes de los medios masivos de comunicación, en aras de una aparente liberación de la sexualidad, se han convertido en otra forma de control sobre el cuerpo femenino. Parafraseando a Basaglia, "una cultura en donde se exalta el aspecto sexual en la vida de una mujer en detrimento de

2 Einsestein, Z., Patriarcado capitalista y feminismo socialista, Sigio.XXI Editores, México, 1984.

3 Mitchel, Juliet, La condicion de la mujer, Extemporáneos, 1974. 
otras cualidades a desarrollar, impide igualmente que esta sexualidad sea verdaderamente suya".

Sabemos que "lo social" tiene incidencia sobre "lo sexual"; pero aún no conocemos con precisión por dónde cruza esa relación; aún desconocemos qué de lo social está regulando la representación de la sexualidad que tiene la mujer. Con esta inquietud nos planteamos dos aspectos por desarrollar en este trabajo, a partir de los datos de la EDEPAM: a) nos propusimos, por una parte, investigar acerca de la toma de decisiones que, sobre su propio cuerpo, realiza la mujer (¿qué tanto decide la mujer acerca del hecho de tener relaciones sexuales?, ¿qué tanto decide acerca del número de hijos a tener?, y ¿qué tan diferentes entre sí son las opiniones sobre estas dos decisiones?), y b) por otra parte, nos interesa conocer si existe alguna relación entre las características socioeconómicas de la mujer y su representación de la sexualidad, con el objeto de reflexionar de qué manera inciden las primeras en la segunda.

Para el análisis siguiente seleccionamos dos variables socioeconómicas: escolaridad y lugar de residencia; ${ }^{4}$ dos demográficas: paridad y ex-

4 Seleccionamos este par de variables tanto por su caracter de diferencial por excelencia de los fenomenos demográficos - la reproducción, entre ellos-, como por el interes que tiene para las representaciones sociales de la sexualidad el contexto educativo en el que uno se desenvuelve. Hemos seleccionado dos indicadores para aproximarnos al sentido del término educación: la escolaridad y el periencia en el uso de anticonceptivos; ${ }^{5}$ una de tipo cultural: el conocimiento o percepción sobre aspectos reproductivos, ${ }^{6}$ y una variable básica de control: la edad de la mujer.

\section{Apropiación del cuerpo femenino}

Tratamos de ubicarnos en la manera como la mujer se apropia de su cuerpo, y explora la opinión sobre las decisiones en cuanto a la reproducción y las relaciones sexuales, y hagamos una "primera lectura" de la información generada por la EDEPAM: en los datos observamos que, a medida que aumenta la escolaridad, se incrementa notablemente el porcen-

lugar de residencia. El primero hace referencia al acceso al sistema escolar propiamente dicho, mientras que el segundo lo elegimos pensando en el proceso educativo al que toda persona se encuentra expuesta por vivir en un contexto determinado. El campo educativo, sociologicamente estudiado, traspasa los límites de la educación escolar, pues incluye también la acción que realizan los grupos primarios como la familia y la clase social a la que se pertenece; y los secundarios, entre los que destacan los grupos políticos, culturales y religiosos.

5 Seleccionamos estas variables, ya que la primera puede considerarse como una de las "consecuencias reproductivas" de las relaciones sexuales, mientras que la segunda hace referencia a una acción que se pone en práctica precisamente para tener cierto control sobre la misma reproducción.

6 El conocimiento de su cuerpo, para una mujer, es un elemento muy importante para tomar decisiones en relación a la fecundidad; cuando éste no es lo suficientemente profundo o correcto, existirán obsLáculos para la utilización de medios de regulación de la fecundidad. En un caso extremo podra llevar a una mujer a percibir que no existe la posibilidad de controlar su fecundidad; en otros, a un uso ina. decuado de los anticonceptivos o al rechazo completo de todos o alguno de ellos. 
taje de mujeres que opina que la decisión de cuándo tener relaciones sexuales debe ser solamente de la pareja, y disminuye el de aquéllas que consideran que dicha decisión es del dominio del hombre (cuadro I). Una tendencia análoga, aunque con cambios menos acentuados, se da al indagar sobre el hecho de que para quién es más importante la relación sexual, para el hombre o para la mujer: al aumentar la escolaridad se incrementa el porcentaje que opina que para los dos es igual de importante, y disminuye el que se lo atribuye preferentemente al hombre (cuadro II). Pareciera que a mayor escolaridad la mujer se siente con más dominio sobre su propia sexualidad $y$, por lo mismo, es factible que se involucre más en cualquier decisión que tenga que ver con ella, como puede ser, para los propósitos de nuestra investigación, el caso de la práctica anticonceptiva.

$\mathrm{Al}$ analizar estos datos en dos contextos educativos diferentes, observamos que la decisión conjunta se incrementa en los ámbitos urbanos, aunque no así la valoración por sexo de la importancia de las relaciones sexuales, lo cual podría hacernos pensar que, aunque exista una valoración similar en ambos contextos, las mujeres de zonas rurales se sienten con "menos derecho" (o por lo menos opinan que deben tener menos derecho) para decidir que las mujeres de zonas urbanas. Podríamos pensar, tal vez, que la valoración o la importancia que se le atribuye a las relaciones sexuales en ambos contextos no determina en última instancia la participación en la toma de decisiones en este aspecto.

Los datos por paridad nos muestran a las mujeres con menos experiencia reproductiva (menos hijos nacidos vivos), con una mayor disposición a involucrarse en la decisión de tener relaciones sexuales, así como de considerar a las relaciones sexuales con una importancia similar para ambos miembros de la pareja.

Las mujeres que alguna vez han hecho algo para influir sobre su fecundidad - como podría esperarseson quienes se involucran más en la decisión sobre las relaciones sexuales; casi la mitad de las mujeres nunca usuarias $(46 \%)$ opino que esta decisión debe ser tomada por el hombre, a diferencia de un $26 \%$ en las mujeres alguna vez usuarias que dieron este tipo de respuesta. No es tan importante la diferencia en la preferencia, por sexo, de la importancia de las relaciones sexuales al considerar condición de uso de métodos anticonceptivos.

El nivel de conocimiento sobre aspectos de la reproducción define también una tendencia con relación a la valoración de las relaciones sexuales: mientras más preciso es el conocimiento, la mujer se involucra más en las decisiones; es decir, cuando se sabe que se puede controlar la posibilidad de un embarazo, se incrementa la posibilidad de decidir cuándo tener relaciones sexuales. Podríamos pensar que un mayor nivel de 


\section{CUADRO I}

Distribución porcentual de las mujeres en edad fértil alguna vez unidas de acuerdo a algunas características socioeconómicas y demográficas, según la persona que debe decidir cuándo tener relaciones sexuales

\begin{tabular}{|c|c|c|c|c|c|c|}
\hline \multirow{2}{*}{$\begin{array}{l}\text { Características de la mujer } \\
\text { Total } \\
\end{array}$} & \multicolumn{6}{|c|}{ Personas que deben decidir cuándo tener relaciones sexuales } \\
\hline & $\begin{array}{c}\text { La mujer } \\
7.5\end{array}$ & $\begin{array}{c}\text { El hombre } \\
31.2\end{array}$ & $\begin{array}{c}\text { Ambos } \\
59.6\end{array}$ & $\begin{array}{c}\text { Otros } \\
0.2\end{array}$ & $\begin{array}{c}\text { N.R. } \\
1.5\end{array}$ & $\begin{array}{l}\text { Total } \\
100 \%\end{array}$ \\
\hline \multicolumn{7}{|l|}{ Grupos de edad } \\
\hline 15 a 24 anos & 7.5 & 31.7 & 59.1 & 0.7 & 1.0 & $100 \%$ \\
\hline 25 a 34 años & 7.5 & 28.3 & 63.4 & 0.0 & 0.8 & $100 \%$ \\
\hline 35 y más & 7.4 & 34.0 & 55.9 & 0.2 & 2.5 & $100 \%$ \\
\hline \multicolumn{7}{|l|}{ Nivel de escolaridad } \\
\hline Sin escolaridad & 6.5 & 44.2 & 45.2 & 0.0 & 4.1 & $100 \%$ \\
\hline Primaria incompleta & 9.8 & 43.8 & 44.2 & 0.6 & 1.6 & $100 \%$ \\
\hline Primaria completa & 6.4 & 17.9 & 75.0 & 0.0 & 0.7 & $100 \%$ \\
\hline Secundaria y más & 5.4 & 13.4 & 81.0 & 0.0 & 0.2 & $100 \%$ \\
\hline \multicolumn{7}{|l|}{ Lugar de residencia } \\
\hline Rural & 8.8 & 39.4 & 48.9 & 0.4 & 2.5 & $100 \%$ \\
\hline Urbano & 6.6 & 25.7 & 66.7 & 0.2 & 0.8 & $100 \%$ \\
\hline \multicolumn{7}{|l|}{ Hilos nacidos vivos } \\
\hline Ninguno & 0.4 & 27.2 & 72.4 & 0.0 & 0.0 & $100 \%$ \\
\hline 1 a 2 hijos & 6.7 & 21.3 & 69.7 & 0.7 & 1.6 & $100 \%$ \\
\hline 3 a 4 hijos & 7.9 & 29.3 & 62.0 & 0.0 & 0.8 & $100 \%$ \\
\hline 50 más hijos & 8.8 & 41.9 & 47.2 & 0.0 & 2.1 & $100 \%$ \\
\hline \multicolumn{7}{|l|}{ Condición de uso } \\
\hline Alguna vez usuaria & 7.3 & 26.4 & 65.1 & 0.0 & 1.2 & $100 \%$ \\
\hline Nunca usuaria & 7.9 & 45.8 & 43.0 & 0.9 & 2.4 & $100 \%$ \\
\hline \multicolumn{7}{|l|}{$\begin{array}{l}\text { Siempre que se tiene relaciones } \\
\text { sexuales se embaraza }\end{array}$} \\
\hline Sí & 7.0 & 44.4 & 45.7 & 0.5 & 2.4 & $100 \%$ \\
\hline No & 7.7 & 24.7 & 66.8 & 0.1 & 0.7 & $100 \%$ \\
\hline No sé & 7.2 & 41.7 & 44.9 & 0.0 & 6.2 & $100 \%$ \\
\hline
\end{tabular}

Fuente: Encuesta sobre Determinantes de la Próctica Anticonceptiva, 1988.

1 En el cuestionario se le preguntó a la entrevistada si cada vez que una mujer tiene relaciones sexuales se emboraza, sin dorle mayores especificaciones. 


\section{CUADRO II}

Distribución porcentual de las mujeres en edad fértil alguna vez unidas de acuerdo a algunas características socioeconómicas y demográficas, según la persona para la cual tienen más importancia las relaciones sexuales

\begin{tabular}{|c|c|c|c|c|c|}
\hline \multirow[b]{2}{*}{ Caracteristicas de la mujer } & \multicolumn{5}{|c|}{ Personas para las cuales tienen más Impotancia las relaciones sexuales } \\
\hline & Para la mujer & Para el hombre & $\begin{array}{l}\text { Es igual de } \\
\text { importante }\end{array}$ & N.R. & Total \\
\hline Total & 2.7 & 39.9 & 54.4 & 3.0 & $100 \%$ \\
\hline \multicolumn{6}{|l|}{ Grupos de edad } \\
\hline 15 a 24 años & 2.7 & 39.0 & 56.1 & 2.2 & $100 \%$ \\
\hline 25 a 34 años & 2.9 & 39.7 & 55.1 & 2.3 & $100 \%$ \\
\hline 35 y más & 2.5 & 40.7 & 52.8 & 4.0 & $100 \%$ \\
\hline \multicolumn{6}{|l|}{ Nivel de escolaridad } \\
\hline Sin escolaridad & 2.0 & 39.4 & 49.3 & 9.3 & $100 \%$ \\
\hline Primaria incompleta & 4.0 & 44.4 & 48.3 & 3.3 & $100 \%$ \\
\hline Primaria completa & 1.6 & 40.2 & 58.2 & 0.0 & $100 \%$ \\
\hline Secundaria y más & 1.9 & 33.3 & 64.3 & 0.5 & $100 \%$ \\
\hline \multicolumn{6}{|l|}{ Lugar de residencia } \\
\hline Rurat & 3.7 & 38.6 & 52.5 & 5.2 & $100 \%$ \\
\hline Urbano & 2.0 & 40.8 & 55.7 & 1.5 & $100 \%$ \\
\hline \multicolumn{6}{|l|}{ Hilos nacidos vivos } \\
\hline Ninguno & 0.0 & 34.0 & 64.0 & 2.0 & $100 \%$ \\
\hline 1 a 2 hijos & 2.7 & 33.9 & 61.5 & 1.9 & $100 \%$ \\
\hline 3 a 4 hijos & 1.6 & 41.5 & 55.3 & 1.6 & $100 \%$ \\
\hline 50 más hijos & 4.0 & 44.6 & 46.2 & 5.2 & $100 \%$ \\
\hline \multicolumn{6}{|l|}{ Condición de uso } \\
\hline Alguna vez usuaria & 1.8 & 40.9 & 55.6 & 1.7 & $100 \%$ \\
\hline Nunca usuaria & 5.3 & 37.0 & 50.8 & 6.9 & $100 \%$ \\
\hline \multicolumn{6}{|l|}{$\begin{array}{l}\text { Siempre que se tiene relaciones } \\
\text { sexuales se embaraza }^{2}\end{array}$} \\
\hline $\mathrm{Si}$ & 4.1 & 37.0 & 53.6 & 5.3 & $100 \%$ \\
\hline No & 2.1 & 41.7 & 55.2 & 1.0 & $100 \%$ \\
\hline No sé & 2.9 & 33.7 & 48.9 & 14.4 & $100 \%$ \\
\hline
\end{tabular}

Fuente: Encuesta sobre Determinantes de la Práctica Anticonceptiva, 1988.

2 En el cuestionario se le preguntó a la entrevistada si cada vezque.una mujer tiene relaciones sexuales se embaraza, sin darle mayores especificaciones.

N.A. 41 
conocimiento trae consigo mayor responsabilidad $y$, al incrementarse la responsabilidad, se incrementa la capacidad de decisión. Esto se confirma si lo relacionamos con los datos del cuadro III: las mujeres con menor instrucción, las de zonas rurales y las que poseen menos conocimiento sobre la reproducción, son las que con mayor frecuencia opinan que el número de hijos por tener depende de la voluntad divina o como responsabilidad del hombre, situándose fuera de la posibilidad de influir sobre la decisión.

Si se analizan las respuestas para toda la población, llama la atención el hecho de que sólo un $7.5 \%$ de las mujeres respondió que la decisión sobre las relaciones sexuales debe ser tomada por la mujer, contra un $31 \%$ que se inclina a pensar que esta decisión debe tomarla el hombre; es decir, cuando la mujer opta por alguno de los dos miembros de la pareja, en 4 de cada 5 casos lo hace por el hombre. Lo anterior nos obliga a recordar la representación del cuerpo femenino como "cuerpo-para-otros" en un grupo importante de mujeres.

Ahora bien, ¿qué es lo que sucede cuando revisamos las decisiones sobre el número de hijos por tener?; ¿sigue los mismos patrones que en las relaciones sexuales? Los datos nos muestran algunas diferencias que vale la pena destacar, aunque en general se mantiene la tendencia a incrementar la participación de ambos miembros de la pareja, a medida que aumenta la escolaridad, el tamaño del lugar de residencia y el nivel de conocimiento sobre la reproducción; asi mismo, aumenta la participación de las mujeres mientras menor es su edad y paridad, y en el caso de que tengan en su haber cierta experiencia anticonceptiva (cuadro III).

Una diferencia que vale la pena destacar, en términos de la reproducción y la sexualidad es el hecho de que la mujer se involucra más en las decisiones sobre los hijos que en lo que se refiere a las relaciones sexuales, disminuyendo a la mitad "el derecho del hombre" a decidir sobre el tema e incrementándose, tanto la decisión individual de la mujer como la decisión conjunta de la pareja (cuadros I y III). Quizá esto se deba a que - como señala Juliet Mitchell- en la mujer recaen, con mayor ahinco, las responsabilidades de la educación y socialización de los hijos, mientras que existen "prohibiciones" sobre su sexualidad; por lo tanto, ella siente que tiene más derecho a decidir sobre la reproducción.

Otro elemento que destaca es el nivel de no respuesta, ya que en alguna forma refleja la facilidad con que la mujer puede abordar el tema sobre el que se le interroga: en el caso de las dos decisiones analizadas, se observan niveles similares (1.5 y 1.3 por ciento) para el total de la población, pero los diferenciales entre las categorías extremas son mayores cuando se habla de sexualidad.

Para todas las características analizadas, los valores más altos de no respuesta se observan en las decisiones 
CUADRO III

Distribución porcentual de las mujeres en edad fértil alguna vez unidas de acuerdo a algunas características socioeconómicas y demográficas, según la persona que debe decidir el número de hijos a tener

\begin{tabular}{|c|c|c|c|c|c|c|c|}
\hline \multirow{2}{*}{ Características de la mujer } & \multicolumn{7}{|c|}{ Personas que deben decidir el número de hijos a tener } \\
\hline & La mujer & El hombre & Ambos & $\begin{array}{l}\text { Los que Dios } \\
\text { mande }\end{array}$ & Otros & N.R. & Total \\
\hline Total & 10.5 & 14.2 & 68.5 & 5.2 & 0.3 & 1.3 & $100 \%$ \\
\hline \multicolumn{8}{|l|}{ Grupos de edad } \\
\hline 15 a 24 años & 9.7 & 16.0 & 69.7 & 3.3 & 0.0 & 1.3 & $100 \%$ \\
\hline 25 a 34 años & 7.7 & 14.7 & 71.0 & 5.2 & 0.2 & 1.2 & $100 \%$ \\
\hline 35 y más & 13.9 & 12.6 & 65.4 & 6.2 & 0.6 & 1.3 & $100 \%$ \\
\hline \multicolumn{8}{|l|}{ Nivel de escolaridad } \\
\hline Sin escolaridad & 12.2 & 22.1 & 48.5 & 13.8 & 1.3 & 2.1 & $100 \%$ \\
\hline Primaria incompleta & 13.8 & 20.1 & 57.3 & 7.2 & 0.2 & 1.5 & $100 \%$ \\
\hline Primaria completa & 11.1 & 9.3 & 78.8 & 0.0 & 0.1 & 0.7 & $100 \%$ \\
\hline Secundaria y más & 4.1 & 3.5 & 91.3 & 0.3 & 0.0 & 0.8 & $100 \%$ \\
\hline \multicolumn{8}{|l|}{ Lugar de residencia } \\
\hline Rural & 11.3 & 21.3 & 53.8 & 11.2 & 0.5 & 1.9 & $100 \%$ \\
\hline Urbano & 10.0 & 9.4 & 78.4 & 1.2 & 0.2 & 0.8 & $100 \%$ \\
\hline \multicolumn{8}{|l|}{ Hijos nacidos vivos } \\
\hline Ninguno & 8.6 & 14.9 & 74.2 & 2.3 & 0.0 & 0.0 & $100 \%$ \\
\hline 1 a 2 hijos & 7.6 & 10.3 & 79.2 & 1.9 & 0.1 & 1.3 & $100 \%$ \\
\hline 3 a 4 hijos & 11.1 & 12.8 & 71.3 & 3.5 & 0.3 & 1.0 & $100 \%$ \\
\hline 50 más hijos & 13.3 & 18.4 & 56.3 & 9.7 & 0.7 & 1.6 & $100 \%$ \\
\hline \multicolumn{8}{|l|}{ Condición de uso } \\
\hline Alguna vez usuaria & 10.1 & 11.8 & 75.5 & 1.2 & 0.2 & 1.2 & $100 \%$ \\
\hline Nunca usuaria & 11.9 & 21.1 & 47.3 & 17.3 & 0.9 & 1.5 & $100 \%$ \\
\hline \multicolumn{8}{|l|}{$\begin{array}{l}\text { Siempre que se tiene relaciones } \\
\text { sexuales se embaraza }{ }^{3}\end{array}$} \\
\hline Si & 13.7 & 20.8 & 52.9 & 10.4 & 0.3 & 1.9 & $100 \%$ \\
\hline No & 0.1 & 10.7 & 77.6 & 1.6 & 0.4 & 0.6 & $100 \%$ \\
\hline No sé & 11.9 & 20.6 & 41.7 & 20.7 & 0.0 & 5.1 & $100 \%$ \\
\hline
\end{tabular}

Fuente: Encuesta sobre Determinantes de la Práctica Anticonceptiva, 1988.

3 En el cuestionario se le preguntó a la entrevistada si cada vezque una mujer tiene relaciones sexuales se embaraza, sin darle moyores especificaciones.

N.A. 41 
sobre relaciones sexuales, y alcanzan un nivel considerablemente mayor cuando se indaga por la persona para la cual dichas relaciones son más importantes, alcanzando valores cercanos al $10 \%$ entre mujeres sin estudios, del $7 \%$ entre nunca usuarias, ligeramente superiores al $5 \%$ entre mujeres que residen en zonas rurales que han tenido 5 hijos o más y entre aquéllas con un conocimiento erróneo sobre la posibilidad de embarazo cada vez que se tienen relaciones sexuales.

Si consideramos que la no respuesta nos indica dificultad para hablar o expresarse sobre el tema, podemos pensar en dos planteamientos hipotéticos: el no hablar sobre el tema puede indicar la existencia de cierta represión para tocar asuntos concernientes a la sexualidad cuando se cuestionan abiertamente; o bien, el no hablar acerca de ellos puede indicar dificultad para "verbalizarlos" debido a que no es del todo consciente la representación que se tiene de la relación sexual o, simplemente, no se siente con derecho $\longrightarrow$ no se tiene la costumbrede involucrarse en tales asuntos.

Tomando en cuenta los objetivos de esta mesa, nos detendremos finalmente a indagar por la representación que de la sexualidad tienen las mujeres, dejando para otro momento su valoración (o representación) de la maternidad, aunque habrá referencias obligadas, como veremos a continuación.

Dividamos la exposición final de esta revisión en tres partes: a) razón por la cual las relaciones sexuales son más importantes para la mujer o para el hombre (desde el puntode vista dela mujer);b) nivel de importancia de las relacionessexualesen la representación de las mujeres; y c) razón de la importancia de las relaciones sexuales para aquellas mujeres que utilizan el término "importante" en su respuesta.

\section{a) Importancia diferencial por sexo de las relaciones sexuales}

La cuarta parte de la población entrevistada, que considera más importante las relaciones sexuales para la mujer, las identifica como la forma de embarazarse, y esto es más frecuente entre mujeres jóvenes (39.8\%), mujeres con menor escolaridad (40.9\%), en zonas rurales $(32.5 \%)$ y en las mujeres que nunca han usado anticonceptivos (39.7\%). En la contraparte, las de más escolaridad, las de zonas urbanas y las que alguna vez han hecho algo para regular su fecundidad, así como las más jovenes, las de menos paridad y las de mejor nivel de conocimiento sobre la reproducción, le asocian un valor afectivo a tales relaciones (cuadro IV). ${ }^{7}$

Si recordamos que el primer grupo de mujeres -con menor escolari-

7 Las categorlas de respuesta, que se analizan en los cuadros IV, V y VII, se seleccionaron por ser las de mayor frecuencia para toda la población en general, aunque esto no siempre se cumpla para cada categorfa de las variables analizadas: es por ello que el rubro de otros lega a tener valores tan altos como los observados. La idea de esta agrupación es analizar que sucede con algunas categorias de respuesta para los diferentes subgrupos poblacionales y de ninguna manera se pretende presentar un desglose exhaustivo del tipo de respucstas encontradas. 


\section{CUADRO IV}

Distribución porcentual de las mujeres en edad fértil alguna vez unidas de acuerdo a algunas características socioeconómicas y demográficas, según la razón por la que las relaciones sexuales son más importantes para la mujer ${ }^{4}$

\begin{tabular}{|c|c|c|c|c|c|c|c|}
\hline \multirow[b]{2}{*}{$\begin{array}{c}\text { Características } \\
\text { de la mujer }\end{array}$} & \multicolumn{7}{|c|}{ Razón de la importancia preferencial para la mujer } \\
\hline & $\begin{array}{c}\text { Es la forma } \\
\text { embarazarse }\end{array}$ & $\begin{array}{c}\text { Porque } \\
\text { queda emba- } \\
\text { razada }\end{array}$ & $\begin{array}{c}\text { Porque desea, } \\
\text { necesita o las } \\
\text { disfruta más }\end{array}$ & $\begin{array}{l}\text { Porque tiene ma- } \\
\text { yor valor afecti- } \\
\text { vo para ella }\end{array}$ & Otros & $\begin{array}{c}\text { No sé } \\
0 \\
\text { N.R. }\end{array}$ & Total \\
\hline Total & 24.4 & 6.6 & 19.5 & 14.2 & 29.8 & 5.5 & $100 \%$ \\
\hline \multicolumn{8}{|l|}{ Grupos de edad } \\
\hline 15 a 24 años & 39.8 & 3.9 & 0.0 & 25.7 & 30.6 & 0.0 & $100 \%$ \\
\hline 25 a 34 años & 13.6 & 0.0 & 16.6 & 18.3 & 39.0 & 12.5 & $100 \%$ \\
\hline 35 y más & 29.4 & 15.8 & 33.1 & 3.4 & 18.3 & 0.0 & $100 \%$ \\
\hline \multicolumn{8}{|l|}{ Nivel de escolaridad } \\
\hline Sin escolaridad & 40.9 & 0.0 & 59.1 & 0.0 & 0.0 & 0.0 & $100 \%$ \\
\hline Primaria incompleta & 23.6 & 11,4 & 10.9 & 0.0 & 44.5 & 9.6 & $100 \%$ \\
\hline Primaria completa & 18.0 & 0.0 & 17.9 & 50.0 & 14.1 & 0.0 & $100 \%$ \\
\hline Secundaria y más & 19.9 & 0.0 & 20.2 & 46.1 & 13.8 & 0.0 & $100 \%$ \\
\hline \multicolumn{8}{|l|}{ Lugar de residencia } \\
\hline Rural & 32.5 & 0.0 & 19.9 & 5.6 & 32.1 & 9.9 & $100 \%$ \\
\hline Urbano & 14.4 & 0.0 & 19.0 & 25.1 & 26.7 & 0.0 & $100 \%$ \\
\hline \multicolumn{8}{|l|}{ Hijos nacidos vivos } \\
\hline Ninguno? & - & $\cdot$ & - & - & - & $\cdot$ & $100 \%$ \\
\hline 1 a 2 hijos & 31.8 & 2.5 & 6.6 & 33.6 & 7.4 & 18.1 & $100 \%$ \\
\hline 3 a 4 hijos & 7.3 & 0.0 & 22.1 & 24.2 & 46.4 & 0.0 & $100 \%$ \\
\hline 50 más hijos & 25.6 & 11.0 & 26.1 & 0.0 & 37.3 & 0.0 & $100 \%$ \\
\hline \multicolumn{8}{|l|}{ Condición de uso } \\
\hline Alguna vez usuaria & 10.1 & 3.1 & 20.3 & 27.6 & 38.9 & 0.0 & $100 \%$ \\
\hline Nunca usuaria & 39.7 & 10.3 & 18.7 & 0.0 & 19.9 & 11.4 & $100 \%$ \\
\hline
\end{tabular}


Continúa CUADRO IV

\begin{tabular}{|c|c|c|c|c|c|c|c|}
\hline $\begin{array}{l}\text { Siempr } \\
\text { relacio } \\
\text { embara }\end{array}$ & & & & & & & \\
\hline SI & 33.6 & 15.4 & 21.2 & 6.5 & 23.3 & 0.0 & $100 \%$ \\
\hline No & 17.9 & 0.0 & 20.6 & 22.6 & 38.9 & 0.0 & $100 \%$ \\
\hline No sé & 15.6 & 0.0 & 0.0 & 0.0 & 0.0 & 84.4 & $100 \%$ \\
\hline
\end{tabular}

Fuente: Encuesta sobre Determinantes de la Próctica Anticonceptiva, 1988.

4 Solo se incluyen mujeres que declararon que las relaciones sexuales son más importantes para ellas. 5 Valoración positiva del embarazo y la maternidad.

6 Valoración negativa del embarazo o la matemidad.

7 No hay mujeres que sin hijos hayan declarado que para la mujer es más importante.

8 En el cuestionario se le preguntó a la entrevistada si cada vez que una mujer tiene relaciones sexuales se embaraza, sin darle mayores especificaciones.

dad, en zonas rurales y mujeres que nunca han usado anticonceptivoses también en el que se observa menor participación en las decisiones, podemos decir que existe relación entre el hecho de decidir menos sobre la reproducción y la sexualidad, y cierta representación de la sexualidad ligada al embarazo. El valorar la relación sexual, no como un goce o algo placentero en sí mismo sino como la posibilidad de embarazarse, nos lleva a reflexionar hasta qué punto el cuerpo de la mujer se encuentra marcado por la función materna en ciertos grupos poblacionales.

Podríamos pensar también que las mujeres que opinan que la relación sexual es importante porque representa la posibilidad del embarazo, valoran más la maternidad como un objetivo de vi 'a de primer orden, o quizá como el único, en contraparte con las mujeres de más escolaridad en quienes probablemente la maternidad sea un objetivo muy importante, pero no el único en su vida.

En el caso del hombre, es otra la "visión" que tienen las mujeres, ya que las tres cuartas partes reconocen que al hombre le son más importantes las relaciones sexuales porque por naturaleza y temperamento le son "más necesarias" (cuadro V). En este caso son las de más escolaridad, las de zonas urbanas, las alguna vez usuarias y las de mejor conocimiento sobre la reproducción, las que declararon en mayor medida, "el temperamento y naturaleza del hombre" como razón de la mayor importancia; sin embargo, nuevamente son las de menos escolaridad, las de áreas rurales, las nunca usuarias y las de menor conocimiento reproductivo, las que no tienen "una razón concreta" para explicar su elección entre hombre y mujer. ¿Será lo que decía 
CUADRO V

Distribución porcentual de las mujeres en edad fértil alguna vez unidas de acuerdo a algunas características socioeconómicas y demográficas, según la razón por la cual las relaciones sexuales son más importantes para el hombre (según la percepción de la mujer) $^{9}$

\begin{tabular}{|c|c|c|c|c|c|}
\hline \multirow[b]{2}{*}{ Caracteristicas de la mujer } & \multicolumn{5}{|c|}{ Razón de la importancia preferencial para el hombre } \\
\hline & $\begin{array}{c}\text { El lo necesita más } \\
\text { por su } \\
\text { temperamento, } \\
\text { por su naturaleza }\end{array}$ & $\begin{array}{c}\text { La mujer se deahoga } \\
\text { con la menstruación y } \\
\text { el hombre con las rela- } \\
\text { ciones sexuales }\end{array}$ & Otros & $\begin{array}{c}\text { No sé } \\
0 \\
\text { N.R. }\end{array}$ & Total \\
\hline Total & 75.1 & 4.7 & 14.3 & 5.9 & $100 \%$ \\
\hline \multicolumn{6}{|l|}{ Grupos de edad } \\
\hline 15 a 24 años & 72.8 & 4.1 & 14.6 & 8.5 & $100 \%$ \\
\hline 25 a 34 años & 74.6 & 3.1 & 14.3 & 8.0 & $100 \%$ \\
\hline 35 y más & 76.6 & 6.6 & 14.2 & 2.6 & $100 \%$ \\
\hline \multicolumn{6}{|l|}{ Nivel de escolaridad } \\
\hline Sin escolaridad & 67.7 & 0.0 & 23.2 & 9.1 & $100 \%$ \\
\hline Primaria incompleta & 74.4 & 3.8 & 13.7 & 8.1 & $100 \%$ \\
\hline Primaria completa & 81.0 & 4.7 & 12.9 & 1.4 & $100 \%$ \\
\hline Secundaria y más & 76.9 & 10.3 & 9.9 & 2.9 & $100 \%$ \\
\hline \multicolumn{6}{|l|}{ Lugar de residencia } \\
\hline Rural & 67.3 & 1.9 & 18.4 & 12.4 & $100 \%$ \\
\hline Urbano & 80.0 & 6.4 & 11.8 & 1.8 & $100 \%$ \\
\hline \multicolumn{6}{|l|}{ Hijos nacidos vivos } \\
\hline Ninguno & 73.0 & 0.0 & 17.9 & 9.1 & $100 \%$ \\
\hline 1 a 2 hijos & 70.9 & 5.0 & 17.0 & 7.1 & $100 \%$ \\
\hline 3 a 4 hijos & 79.1 & 4.7 & 12.7 & 3.5 & $100 \%$ \\
\hline 50 más hijos & 75.0 & 4.9 & 13.5 & 6.6 & $100 \%$ \\
\hline \multicolumn{6}{|l|}{ Condición de uso } \\
\hline Alguna vez usuaria & 78.5 & 5.4 & 12.8 & 3.3 & $100 \%$ \\
\hline Nunca usuaria & 63.8 & 2.4 & 19.1 & 14.7 & $100 \%$ \\
\hline
\end{tabular}

N.A. 41 
Continúa CUADRO V

\begin{tabular}{llllll}
\hline Slempre que se tiene relaciones $^{\text {sexuales se embaraza }^{10}}$ & & & & & \\
Si & 66.5 & 3.6 & 21.4 & 8.5 & $100 \%$ \\
No & 79.0 & 5.5 & 11.1 & 4.4 & $100 \%$ \\
No sé & 65.5 & 0.0 & 22.0 & 12.5 & $100 \%$ \\
\hline
\end{tabular}

Fuente: Encuesta sobre Determinantes de la Próctica Anticonceptiva, 1988.

9 Se incluyen únicamente a las mujeres que contestaron que las relaciones sexuales son más importantes para el hombre.

10 En el cuestionario se le preguntó a la entrevistada si cada vez que una mujer tiene relaciones sexuales se emboraza, sin darle mayores especificaciones.

Vieira Pinto ${ }^{8}$ sobre la dificultad de la "preocupación" en los subgrupos poblacionales que viven más en el nivel de subsistencia, y que no acostumbran "planear" o "tomar distancia" de su situación actual?; ¿'será realmente una falta de representación o "simplemente" una representación reprimida?

b) Importancia de las relaciones sexuales para la mujer

Con el fin de evitar que la mujer hable de la sexualidad únicamente en contra-posición con la sexualidad masculina, concluimos nuestro cuestionario de la encuesta indagando por la importancia que le atribuye la mujer entrevistada a las relaciones sexuales en el nivel personal. Esta pregunta abre un abanico de significados que involucran opiniones,

8 Vieira Pinto, A., El pensamiento crilico en demografia, Santiago de Chile, Centro Latinoamericano de Demografia, 1973. creencias, demandas afectivas y valores, que deposita la mujer en la relación sexual viviendo en pareja. Es precisamente la respuesta a esta pregunta en donde mejor se puede apreciar la subjetividad y los valores culturales ligados al ejercicio de la sexualidad.

El $57 \%$ de las mujeres valoran positivamente las relaciones sexuales, el $21 \%$ no las descartan de manera definitiva, a un 2.5\% "le dan lo mismo" o no sabe qué responder, el $2 \%$ no quiso contestar y un $17.6 \%$ declaró abiertamente que no le resultan importantes (cuadro VI).

Entre las mujeres que las descartan deben destacarse las de menor escolaridad, las de zonas rurales, las de mayor edad y paridad, y las nunca usuarias. Sin embargo, no siempre se presenta un diferencial en sentido opuesto al analizar la categoría de "importante", ya que son la escolaridad, la paridad y la edad, las únicas características que marcan diferencias importantes entre los grupos extremos. 


\section{CUADRO VI}

Distribución porcentual de las mujeres en edad fértil alguna vez unidas de acuerdo a algunas características socioeconómicas y demográficas, según la importancia que le atribuyen a las relaciones sexuales

\begin{tabular}{|c|c|c|c|c|c|c|c|}
\hline \multirow[b]{2}{*}{$\begin{array}{c}\text { Características } \\
\text { de la mujer }\end{array}$} & \multicolumn{7}{|c|}{ Nivel de importancia de las relaciones sexuales } \\
\hline & $\begin{array}{l}\text { Importante (muy } \\
\text { importante, bueno) }\end{array}$ & $\begin{array}{l}\text { No es tan importan- } \\
\text { te (más o menos) }\end{array}$ & $\begin{array}{l}\text { No es im- } \\
\text { portante }\end{array}$ & $\begin{array}{l}\text { Me da } \\
\text { igual }\end{array}$ & No sé & N.R. & Total \\
\hline Total & 57.0 & 21.0 & $\uparrow 7.6$ & 1.5 & 1.0 & 1.9 & $100 \%$ \\
\hline \multicolumn{8}{|l|}{ Grupos de edad } \\
\hline 15 a 24 años & 64.0 & 20.5 & 12.3 & 0.4 & 0.8 & 2.0 & $100 \%$ \\
\hline 25 a 34 años & 56.8 & 23.0 & 15.2 & 2.2 & 1.1 & 1.7 & $100 \%$ \\
\hline 35 y más & 53.8 & 19.1 & 22.6 & 1.3 & 1.0 & 2.2 & $100 \%$ \\
\hline \multicolumn{8}{|l|}{ Nivel de escolaridad } \\
\hline Sin estudios & 56.2 & 19.1 & 17.9 & 1.5 & 3.0 & 2.3 & $100 \%$ \\
\hline Primaria incompleta & 46.3 & 21.0 & 27.5 & 1.5 & 0.7 & 3.0 & $100 \%$ \\
\hline Primaria completa & 63.6 & 21.7 & 11.8 & 1.5 & 0.0 & 1.4 & $100 \%$ \\
\hline Secundaria y más & 68.7 & 21.7 & 6.6 & 1.6 & 0.8 & 0.6 & $100 \%$ \\
\hline \multicolumn{8}{|l|}{ Lugar de residencia } \\
\hline Rural & 56.4 & 17.4 & 19.6 & 1.6 & 1.3 & 3.7 & $100 \%$ \\
\hline Urbano & 57.4 & 23.4 & 16.2 & 1.4 & 0.8 & 0.8 & $100 \%$ \\
\hline \multicolumn{8}{|l|}{ Hiios nacidos vivos } \\
\hline Ninguno & 67.7 & 23.5 & 8.1 & 0.7 & 0.0 & 0.0 & $100 \%$ \\
\hline 1 a 2 hijos & 64.7 & 19.4 & 12.0 & 1.7 & 0.7 & 1.5 & $100 \%$ \\
\hline 3 a 4 hijos & 57.1 & 24.6 & 14.5 & 1.5 & 0.5 & 1.8 & $100 \%$ \\
\hline 50 más hijos & 48.7 & 19.2 & 26.2 & 1.4 & 1.7 & 2.8 & $100 \%$ \\
\hline \multicolumn{8}{|l|}{ Condición de uso } \\
\hline Alguna vez usuaria & 57.7 & 22.5 & 16.5 & 1.6 & 0.6 & 1.1 & $100 \%$ \\
\hline Nunca usuaria & 54.8 & 16.5 & 20.7 & 1.3 & 2.0 & 4.7 & $100 \%$ \\
\hline \multicolumn{8}{|c|}{ Siempre que se tiene relaciones sexuales se embaraza ${ }^{11}$} \\
\hline $\mathrm{Si}$ & 57.8 & 14.4 & 21.9 & 1.1 & 1.2 & 3.6 & $100 \%$ \\
\hline No & 57.7 & 23.8 & 15.5 & 1.8 & 0.6 & 0.6 & $100 \%$ \\
\hline No sé & 45.4 & 21.2 & 19.8 & 0.0 & 4.4 & 9.2 & $100 \%$ \\
\hline
\end{tabular}

Fuente: Encuesta sobre Determinantes de la Práctica Anticonceptiva, 1988.

1 En el cuestionario se le preguntó a la entrevistado si cada vez que una mujer tiene relaciones sexuales se embaraza, sin darle mayores especificaciones. 
c) Sentido de la importancia de las relaciones sexuales

Los datos del último cuadro nos muestran diferentes procesos de racionalización y "verbalización" entre distintos grupos poblacionales: es aquí donde se comprueba la riqueza y ambigüedad del lenguaje que utilizamos para "hablar" de nuestras representaciones sociales. Mientras que para las mujeres con pocos estudios, en zonas rurales y sin experiencia anticonceptiva, la importancia de las relaciones sexuales se sustenta por razones ("más elementales") como el ser agradable, estar bien o estar contenta, entre las mujeres con más estudios, que viven en zonas urbanas y que alguna vez han regulado su fecundidad, hay un mayor espacio para hablar de comunicación en la pareja $\mathrm{e}$, incluso, de una necesidad fisiológica y un desahogo corporal (cuadro VII). A menor paridad se incrementa esta última categoría, algo que no sucede entre los distintos grupos de edad.

\section{A MANERA DE CONCLUSIONES}

El trabajo no permite en este momento generar conclusiones definitivas, si bien nos brinda elementos para plantear algunas reflexiones finales. Tocar el tema de las representaciones sociales es situarse en el terreno de lo subjetivo. Cuando uno se coloca en este ámbito, y pretende realizar análisis, éste se complica, se torna de gran complejidad; pero coincidimos con George Devereux cuando dice que en las ciencias sociales sólo se logra la objetividad cuando se incluye el estudio de la subjetividad. En nuestro trabajo hemos presentado un primer ejercicio, un primer acercamiento al estudio de la representación social acerca de la sexualidad femenina, a partir de la información generada por una encuesta (EDEPAM), en la que la sexualidad se concibe como una variable cultural que incide en la práctica anticonceptiva.

La relación entre anticoncepción y sexualidad es sumamente compleja, puesto que lo sexual no deviene directamente una conducta regulativa frente a la reproducción. Ni la sexualidad puede reducirse sólo al coito, ni la práctica anticonceptiva puede concebirse únicamente como la acción que evita un embarazo. Tanto un concepto como otro se encuentran nutridos de una gran cantidad de componentes sociales y culturales, que se interponen o que se convierten en mediaciones entre los dos fenómenos. ${ }^{9}$ Sin embargo, no dejamos de preguntarnos si en realidad ciertos grupos de mujeres "deciden menos", o si están acostumbradas a sentir que deben decidir menos. ¿Será que más que no tener claras las opciones temáticamente hablando, que

9 Rivera, G., Figueroa, J.G., Cervantes, A., Practica anticonceptiva y sexualidad en México: un andlisis exploratorio, Trabajo presentado en la XIV Reunion Nacional del Consejo Nacional para la Enseñanza e Investigación en Psicologia, S.L.P., Mayo de 1989. 
CUADRO VII

Distribución porcentual de las mujeres en edad fértil alguna vez unidas de acuerdo a algunas características socioeconómicas y demográficas, según la razón de la importancia que le atribuyen a las relaciones sexuales 12

\begin{tabular}{|c|c|c|c|c|c|c|c|}
\hline \multirow[b]{2}{*}{$\begin{array}{l}\text { Características } \\
\text { de la mujer }\end{array}$} & \multicolumn{7}{|c|}{ Razón de la importancia de las relaciones sexuales } \\
\hline & $\begin{array}{l}\text { Algo agra- } \\
\text { dable o sa- } \\
\text { tisfactorio }\end{array}$ & $\begin{array}{l}\text { Manifestación } \\
\text { de afecto, cari- } \\
\text { ño, a mor }\end{array}$ & $\begin{array}{l}\text { Para es- } \\
\text { tar bien, } \\
\text { contenta }\end{array}$ & $\begin{array}{l}\text { Para unir, co } \\
\text { municar, comple- } \\
\text { mentar a la pareja }\end{array}$ & $\begin{array}{l}\text { Necesidad fisio- } \\
\text { lógica, desaho- } \\
\text { go corporal }\end{array}$ & Otros & Total \\
\hline Total & 12.7 & 14.0 & 9.4 & 23.0 & 17.0 & 23.9 & $100 \%$ \\
\hline \multicolumn{8}{|l|}{ Grupos de edad } \\
\hline 15 a 24 años & 20.2 & 11.3 & 8.1 & 22.5 & 15.3 & 22.6 & $100 \%$ \\
\hline 25 a 34 años & 9.7 & 14.6 & 11.1 & 22.8 & 18.3 & 23.5 & $100 \%$ \\
\hline 35 y más & 11.5 & 14.9 & 8.3 & 23.4 & 16.6 & 25.3 & $100 \%$ \\
\hline \multicolumn{8}{|l|}{ Nivel de escolaridad } \\
\hline Sin estudios & 16.5 & 14.8 & 13.4 & 13.4 & 9.2 & 32.7 & $100 \%$ \\
\hline Primaria incompleta & 17.1 & 11.7 & 11.9 & 21.2 & 10.9 & 27.2 & $100 \%$ \\
\hline Primaria completa & 9.2 & 12.9 & 8.2 & 20.6 & 24.1 & 25.0 & $100 \%$ \\
\hline Secundaria y más & 8.5 & 16.6 & 5.5 & 31.7 & 22.5 & 15.2 & $100 \%$ \\
\hline \multicolumn{8}{|l|}{ Lugar de residencia } \\
\hline Rural & 17.1 & 11.1 & 13.4 & 19.9 & 10.5 & 28.0 & $100 \%$ \\
\hline Urbano & 9.8 & 15.8 & 6.7 & 24.9 & 21.3 & 21.5 & $100 \%$ \\
\hline \multicolumn{8}{|l|}{ Hijos nacidos vivos } \\
\hline Ninguno & 19.9 & 5.3 & 5.0 & 30.6 & 18.6 & 20.6 & $100 \%$ \\
\hline 1 a 2 hijos & 12.2 & 17.3 & 7.6 & 23.4 & 17.6 & 21.9 & $100 \%$ \\
\hline 3 a 4 hijos & 9.0 & 12.1 & 9.2 & 27.3 & 20.2 & 22.2 & $100 \%$ \\
\hline 5 o más hijos & 15.4 & 13.5 & 12.5 & 16.9 & 13.0 & 28.7 & $100 \%$ \\
\hline \multicolumn{8}{|l|}{ Condición de uso } \\
\hline Alguna vez usuaria & 10.9 & 14.3 & 7.5 & 24.4 & 20.1 & 22.8 & $100 \%$ \\
\hline Nunca usuaria & 18.5 & 12.8 & 15.3 & 18.2 & 7.0 & 28.2 & $100 \%$ \\
\hline \multicolumn{8}{|c|}{ Sjempre que se tiene relaciones sexuales se embaraza ${ }^{13}$} \\
\hline Si & 19.4 & 17.4 & 12.3 & 16.4 & 8.4 & 26.1 & $100 \%$ \\
\hline No & 8.5 & 13.1 & 8.0 & 26.2 & 21.0 & 23.2 & $100 \%$ \\
\hline No sé & 31.1 & 5.0 & 11.3 & 16.9 & 11.6 & 24.1 & $100 \%$ \\
\hline
\end{tabular}

N.A. 41 
en justicia les corresponden, no se sienten con derecho a tener tales opciones? ¿Será que el otro grupo de mujeres sabe que tiene el derecho, y aunque no lo ejercite, se siente mejor al decir que lo utiliza?

Los planteamientos anteriores nos llevan a afirmar que no pueden pretenderse explicaciones universales en el estudio de la representación de la sexualidad. Consideramos que la sexualidad es un área "vicarial" en donde confluyen la historia personal y las representaciones sociales.

Hasta aquí sabemos que la representación de la sexualidad, la función materna y la práctica anticonceptiva, guardan entre sí una relación compleja, en la que no es del todo fácil determinar causas y efectos. Podemos decir que la posibilidad del embarazo matiza de manera significativa la representación social de la sexualidad en ciertos grupos de mujeres: las de menos escolaridad, las de zonas rurales, las que no cuentan con experiencia en el uso de anticonceptivos y las que tienen un conocimiento erróneo sobre algunos elementos de la reproducción. Los niveles de no respuesta en materia de sexualidad que se observan en estos grupos quizá se deban a que tienen menos posibilidades de "verbalización" sobre el tema. Vale la pena reflexionar acerca de los espacios con que cuentan estos grupos de mujeres para poder expresar, verbalmente, las inquietudes, temores y deseos en torno a su propio cuerpo y esclarecer un poco las con- tradicciones que encierra la identidad femenina.

Encontramos que a mayor escolaridad, a mayor tamaño de la localidad de residencia, a menor edad y paridad, y a mayor conocimiento sobre elementos de la reproducción, se incrementa la práctica (o por lo menos el discurso) de "la igualdad de derechos" en las decisiones acerca de la sexualidad y la reproducción, pero no sólo la escolaridad lleva a un incremento importante en la revaloración de las relaciones sexuales.

Parecería como si la toma de decisiones en torno al propio cuerpo, no estuviera determinada por las mismas características que determinan la valoración o importancia que se atribuye a la relación sexual. Si leemos la capacidad de tomar decisiones sobre el propio cuerpo, como una manera de apropiarse del mismo, podríamos decir que la única variable que marca diferencias importantes en este aspecto es la escolaridad.

Vale la pena preguntarse cuáles son aquellos contenidos que se transmiten por medio de la "educación escolar" y que no se obtienen más que por ese medio. Conviene cuestionar si es posible adquirir estos contenidos por otras vías: una posible alternativa es abrir espacios en los medios de comunicación a través de los cuales las mujeres pudieran "re-pensar" y "pre-ocuparse" de su propia sexualidad, en aras de una sexualidad ligada más al goce y a sus necesidades afectivas y corporales, y no únicamente a la funcion materna. 
Sabemos que los medios masivos de comunicación son "Ia otra escuela"; creemos que un cambio en la representación social de la mujer, que en ellos se transmite, incidirá en un cambio en el grado de conciencia de la sexualidad, y posibilitaría que la mujer viviera su cuerpo de otra manera. Surge la pregunta en términos de las repercusiones que esta posibilidad podría tener, tanto en el nivel de la práctica anticonceptiva como en la representación social de lo que significa ser mujer. $\pi$

\section{BIBLIOGRAFIA}

BASAGLIA, F. et al., "La mujer y la locura", Antipsiquiatría y Politica, Extemporáneos, 1978, pp. 159-180.

CATALA, M., Reflexiones desde un cuerpo de mujer, Anagrama, 1984.

EISENSTEIN, Z., Patriarcado capitalista y femenismo socialista, Siglo XXI Editores México, 1984.
FIGUeroA, J. Cervantes, A., Palma, Y. y Aparicio, R. Determinantes de la práctica anticonceptiva en México, Documento Metodológico, DGPF. Secretaría de Salud, México, 1988.

FOUCAULT, M., Historia de la sexualidad, t. I, México, Siglo XXI Editores, 1987.

KAES, R., El aparato psíquico grupal, GEDISA, 1977.

LANGER, M., Maternidad y sexo, Paidos España, 1988.

MITCHEL, J., La condición de la mujer, Extemporáneos, 1974.

MOSCOVICI, S., La psicología social, una disciplina en movimiento., París, La Haya, (s.f.).

RIVERA, G., Figueroa. J.G., Cervantes, A., Práctica anticonceptiva y sexualidad en México: un análisis exploratorio, Trabajo presentado en la XIV Reunión Nacional del Consejo Nacional para la Enseñanza e Investigación en Psicología, S.L.P., Mayo de 1989.

TORREGROSA, J. et al., Teorfa e investigacion en la psicologia social actual, Madrid, Instituto de la Opinión Pública, 1974. 